\title{
Evaluation of blood vessel injury, oxidative stress and circulating inflammatory factors in an L-NAME-induced preeclampsia-like rat model
}

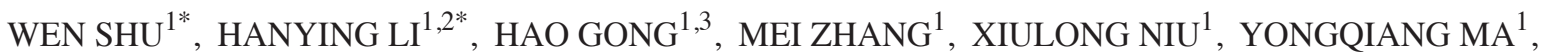 \\ XIN ZHANG $^{1}$, WEI CAI ${ }^{1}$, GUOHONG YANG $^{1}$, MAOTI WEI $^{1}$, NING YANG $^{1}$ and YUMING LI ${ }^{1}$ \\ ${ }^{1}$ Tianjin Key Laboratory of Cardiovascular Remodeling and Target Organ Injury, Pingjin Hospital Heart Center, \\ Tianjin 300162; ${ }^{2}$ Graduate School of Medicine, Tianjin Medical University, Tianjin 300070; \\ ${ }^{3}$ Jinzhou Medical University, Jinzhou, Liaoning 121001, P.R. China
}

Received November 21, 2017; Accepted April 13, 2018

DOI: $10.3892 /$ etm.2018.6217

\begin{abstract}
Preeclampsia is a pregnancy-specific disease characterized by hypertension as well as proteinuria after the 20th week of pregnancy. Animal models are effective tools for studying the pathogenesis, diagnostic criteria and treatment methods of preeclampsia. The present study sought to establish and evaluate a preeclampsia-like Sprague Dawley (SD) rat model using N-nitro-L-arginine methyl ester (L-NAME). Rats were randomly assigned to 7 groups ( $\mathrm{n}=10$ in each): Control rats and rats treated with low-dose L-NAME (40 mg/kg body weight/day) starting from gestational day (GD) 9 , medium-L-NAME ( $75 \mathrm{mg} / \mathrm{kg}$ body weight/day) starting from GD 9 (9D ML group), high-dose L-NAME (125 mg/kg body weight/day) starting from GD 9, low-dose L-NAME starting from GD 10, medium-dose L-NAME starting from GD 10 and high-dose L-NAME starting from GD 10. Blood pressure (BP), 24-h proteinuria, fetal intrauterine growth, histopathological changes, the plasma soluble fms-like tyrosine kinase-1 (sFlt-1)/placental growth factor (PLGF) ratio and cytokine levels were evaluated. Elevated BP, increased urinary albumin excretion, severe endotheliosis, mesangial expansion and increased sFlt-1/PLGF ratios were observed in the experimental groups compared with the control group $(\mathrm{P}<0.05)$, particularly in the 9D ML group. The results of the present study may optimize the conditions of the previously
\end{abstract}

Correspondence to: Professor Ning Yang or Professor Yuming Li, Tianjin Key Laboratory of Cardiovascular Remodeling and Target Organ Injury, Pingjin Hospital Heart Center, 220 Cheng-Lin Road, Tianjin 300162, P.R. China

E-mail: yangningzxdl@sina.com

E-mail: cardiolab@live.com

\section{${ }^{*}$ Contributed equally}

Key words: preeclampsia, hypertension, proteinuria, oxidative stress, nitric oxide established L-NAME-induced preeclampsia SD rat model and aid further study into the pathogenesis of preeclampsia.

\section{Introduction}

Preeclampsia is a hypertensive disorder of pregnancy that is characterized by systolic blood pressure (SBP) $\geq 140 \mathrm{mmHg}$ or diastolic BP $\geq 90 \mathrm{mmHg}$, as well as proteinuria ( $\geq 0.3 \mathrm{~g} / 24 \mathrm{~h})$ after the 20th week of pregnancy (1-4). It is a multisystem syndrome, and the maternal and neonatal morbidity in the setting of preeclampsia is significantly greater than that associated with normal pregnancies $(5,6)$.

Animal models are effective tools for studying the pathogenesis, diagnostic criteria and treatment methods of preeclampsia. It is well known that nitric oxide (NO) in vascular endothelial cells is critical for regulating vascular tension. During normal pregnancy, the cardiac output, blood volume and heart rate are increased; however, the BP typically remains normal or is slightly lower than that prior to pregnancy (7). NO has an important role in the regulation of the cardiovascular system during pregnancy (7). N-nitro-L-arginine methyl ester (L-NAME) is an inhibitor of NO synthase (NOS), and inhibition of NOS has been demonstrated to alleviate hypertension in pregnant rats as well as maintain normal BP levels until delivery (8). Since preeclampsia is associated with vascular endothelial disorders and excessive inflammation (9), it is important to construct a preeclampsia-like animal model that mimics the vascular pathology of preeclampsia to better understand its onset and progression.

It has been proposed that the development of preeclampsia is likely associated with placenta formation (10), particularly with placental dysfunction in early pregnancy $(6,11,12)$. Although the exact mechanisms of preeclampsia remain elusive, vascular dysfunction, oxidative stress and metabolic abnormalities are possible causes of maternal preeclampsia syndrome. In addition, vasoactive substances are considered to be another pathogenic factor for the development of preeclampsia (13). Hypoxia caused by the lack of blood flow in the placenta may induce the release of various vasoactive substances, including anti-angiogenic factors (14). 
Furthermore, a previous study indicated that abnormalities in circulating angiogenic factors have an important role in the pathogenesis of preeclampsia (15). To date, a large number of clinical studies and experimental studies have provided strong evidence that the soluble fms-like tyrosine kinase-1 (sFlt-1)/placental growth factor (PLGF) ratio may be used as a predictor of preeclampsia, particularly early-onset preeclampsia (16). Therefore, the present study aimed to establish an L-NAME-induced rat model of preeclampsia and evaluate vascular injury, oxidative stress and the circulating vasoactive substances in the rats in order to confirm the feasibility of their use as a model of preeclampsia.

\section{Materials and methods}

Reagents. N-nitro-L-arginine methyl ester (L-NAME) was purchased from Sigma-Aldrich (Merck KGaA, Darmstadt, Germany). The bicinchoninic acid (BCA) protein assay kit was acquired from Thermo Fisher Scientific (Waltham, MA, USA). PLGF, sFlt-1, 8-iso-prostaglandin F2 $\alpha$ (8-iso-PGF2 $\alpha$ ) and MDA activity was measured using a commercial kit from Jiancheng Institute (cat. no. A003-1; Nanjing, China). Levels of common inflammatory markers were measured using a cytometric beads assay (LEGENDplex ${ }^{\mathrm{TM}}$ Rat Panel; BioLegend, San Diego, CA, USA).

Animals. A total of 70 female SD rats (weight, 200-220 g; age, 6-7 weeks) were bred at the Laboratory Animal Center of the Academy of Military Medical Sciences of Beijing People's Liberation Army (Beijing, China). Respective parental rats were not subjected to any experiments or treatments prior to breeding. The animals were individually housed at $24^{\circ} \mathrm{C}$ with a humidity of $55 \pm 10 \%$ under a $12 \mathrm{~h}$ light/dark cycle and were provided regular chow and water at libitum. At the age of 8 weeks, the female rats were pair-housed with a male rat. On the morning after mating, clean and wet cotton swabs (rinsed with physiological saline) were gently inserted into the vagina of female rats rotated twice to obtain the vaginal secretions, which were smeared on clean and dry slides and observed under an optical microscope. The presence of sperm in the vaginal smears was indicative of gestational day 0 (GD 0). Rats were randomly assigned to 7 groups, with dosages selected according to a previous study (17): Control rats; rats treated with low-dose L-NAME (40 mg/kg body weight/day) starting from GD 9 (9D LL); rats treated with medium-dose L-NAME (75 mg/kg body weight/day; selected according to a pervious study) (18) starting from GD 9 (9D ML); rats treated with high-dose L-NAME (125 mg/kg body weight/day) starting from GD 9 (9D HL); rats treated with low-dose L-NAME (40 mg/kg body weight/day) starting from GD 10 (10D LL); rats treated with medium-dose L-NAME $(75 \mathrm{mg} / \mathrm{kg}$ body weight/day) starting from GD 10 (10D ML); and rats treated with high-dose L-NAME (125 mg/kg body weight/day) starting from GD 10 (10D HL). Pregnant rats were administered different doses of L-NAME starting on the ninth or tenth day of gestation via subcutaneous injection on the neck back. All treatments were maintained until GD 20. The combination of different dosages and time-points is the core of the present experiment. Multiple injections were applied to maintain the drug effect of L-NAME, resulting in a persistent high BP of pregnant rats and other physiological changes, including abnormal urine protein and endothelial dysfunction $(17,19)$. All experimental animals were euthanized on GD 20.

Ethics statements. The experiments were approved by the ethics committee of the Investigation of Logistics University of the Chinese People's Armed Police Force (Tianjin, China), and the treatment of all 70 animals was compliant with the Public Health Service Policy on the Humane Care and Use of Laboratory Animals of the National Institutes of Health.

Measurement of BP. BP was non-invasively measured during pregnancy on GD 0, 6, 12 and 18 by determining the tail blood volume with a volume pressure-recording sensor and an occlusion tail cuff (CODA System; Kent Scientific, Torrington, CT, USA) (19).

Urine protein quantitation. Rats were placed in metabolic cages for $24 \mathrm{~h}$ for urine collection at baseline (prior to mating) and on GD19. The 24-h urine protein in each group was measured with a BCA protein assay kit (Thermo Fisher Scientific, Inc.).

Tissue collection. Maternal blood was collected on GD 20 by abdominal aorta puncture after the rats were anesthetized with $3 \%$ intraperitoneal pentobarbital sodium $(50 \mathrm{mg} / \mathrm{kg})$. Animals were then sacrificed via exsanguination, which was confirmed following following a complete non-autonomous response to external stimuli. Plasma was prepared by centrifugation at $1,006.2 \mathrm{xg}$ for $20 \mathrm{~min}$ at $4^{\circ} \mathrm{C}$. The fetuses, placentas and kidneys were removed, weighed and then fixed with $4 \%$ paraformaldehyde for histological evaluation. The uterus was cut and the placenta and fetuses were removed quickly. Fetal processing was conducted in accordance with CCAC Guidelines (20). The plasma samples and the remaining placentas and kidneys were stored at $-80^{\circ} \mathrm{C}$ until analysis.

Histological assay. Placenta and kidney specimens were cut into $5-\mu \mathrm{m}$ paraffin sections using a microtome and were stained with hematoxylin and eosin for conventional morphological evaluation under a light microscope (Olympus BX51; Olympus, Tokyo, Japan).

LEGENDplex ${ }^{T M}$. The LEGENDplex ${ }^{\mathrm{TM}}$ Rat Panel is a bead-based multiplex assay panel using fluorescence-encoded beads that are suitable for use on various flow cytometers. Tumor necrosis factor- $\alpha$ (TNF- $\alpha$ ), interleukin-1 $\beta$ (IL-1 $\beta$ ), IL-6, IL-17A and monocyte chemoattractant protein 1 (MCP-1) levels were examined according to methods described in a previous study (21-23). In brief, the standard was prepared by dissolving in $250 \mu \mathrm{l}$ Assay Buffer and 4-fold dilution; Assay Buffer was used as the $\mathrm{C} 0$ standard $(0 \mathrm{pg} / \mathrm{ml})$. Matrix B was added to the samples, and subsequently, Assay Buffer and beads were added to reach a final volume of $100 \mu \mathrm{l}$ per tube. The samples were then mixed and incubated in the dark at room temperature. Subsequently, Streptavidin and R-Phycoerythrin conjugate was added and the sample was mixed. Following centrifugation at a speed of $1,000 \mathrm{x} \mathrm{g}$ for $5 \mathrm{~min}$ at $22^{\circ} \mathrm{C}$, the supernatant was discarded, and the beads were vortexed and analyzed with a flow cytometer. The signal intensity of the microbeads was 
detected using a BD LSRFortessa instrument (BD Biosciences, Franklin Lakes, NJ, USA), and the data were analyzed using LEGENDplex software, version 8.0 (BioLegend).

ELISA. Plasma was separated by centrifuging the blood at $1,006.2 \mathrm{x} \mathrm{g}$ for $20 \mathrm{~min}$ at $4^{\circ} \mathrm{C}$. sFlt-1, PLGF and 8-iso-PFG2 $\alpha$ were measured by quantitative sandwich enzyme immunoassays using commercial ELISA kits.

Thiobarbituric acid reactive substances (TBARS) assay. The supernatant of the tissue homogenate (with PBS) was decanted into glass tubes and mixed with $500 \mu 12 \%$ phosphoric acid. Subsequently, $7 \% \mathrm{H}_{3} \mathrm{PO}_{4}(200 \mu \mathrm{l})$, TBA/butylated hydroxytoluene $(400 \mu \mathrm{l})$ and $1 \mathrm{M} \mathrm{HCl}(200 \mu \mathrm{l})$ were added. The tubes were heated at $100^{\circ} \mathrm{C}$ for $15 \mathrm{~min}$ and then cooled to room temperature. Butanol $(1,500 \mu \mathrm{l})$ was then added to each tube, followed by vortexing. The upper phase of each vial was transferred to triplicate wells of a 96-well plate, and the absorbance (Abs.) was measured at 532 and $600 \mathrm{~nm}$ using a Spectrostarnano plate reader (BMG Labtech, Ortenberg, Germany). The concentration was determined using the following formula: Concentration $(\mathrm{nmol} / \mathrm{mgprot})=[($ Abs.532 $\mathrm{n}$ m-Abs.600 nm)/156] x1,000.

Measurement of serum NO levels. The serum concentration of NO was measured with an NO assay kit (nitrate reductase method; cat. no. A012; Nanjing Jiancheng Bioengineering Institute, Nanjing, China) in accordance with the manufacturer's protocol.

Statistical analysis. All values are expressed as the mean \pm standard deviation. Statistical analysis was performed using STATA version 14.2 (StataCorp LP, College Station, TX, USA). Statistical analyses were performed by one-way analysis of variance (ANOVA) followed by an S-N-K post-hoc test. BP during pregnancy was analyzed by repeated-measures ANOVA. $\mathrm{P}<0.05$ was considered to indicate a statistically significant difference.

\section{Results}

Systolic and mean arterial pressure changes in the pregnant rat. The variation of SBP and MAP during pregnancy in each group is displayed in Fig. 1A and B. Prior to L-NAME administration, no significant differences in SBP and mean arterial pressure (MAP) were present among the 7 groups of rats (the 9D LL, 9D ML, 9D HL, 10D LL, 10D ML, 10D HL and normal pregnancy groups). The SBP of the L-NAME groups steadily increased with L-NAME treatment, and the SBP of the 9D ML, 9D HL and 10D ML groups was slightly increased during late gestation (Fig. 1A). Of note, the SBP of the L-NAME-treated pregnant rats in the 9D ML group was significantly higher than that of rats in the control group $(\mathrm{P}<0.01)$ and the other L-NAME-treated groups $(\mathrm{P}<0.05)$. The MAP in the L-NAME-treated groups was higher than that in the control group $(\mathrm{P}<0.05)$, and no significant difference in MAP was present among the groups treated with L-NAME (Fig. 1B).

The rats in all groups except the 9D ML group exhibited patterns that are normal during pregnancy, with a decrease in SBP and MAP during the late stage. However, the rats in the 9D ML group did not exhibit this drop in BP, as their BP steadily increased during pregnancy.

Changes in kidney structure and function in the pregnant rats injected with L-NAME. Renal histology of the pregnant rats in the L-NAME-treated groups exhibited significant changes that are consistent with those observed in human preeclampsia patients. The kidneys of the pregnant L-NAME-treated rats exhibited an increased glomerular area, capillary structure disorder and abnormal protein cast. Representative images for the control, 9D ML and 10D ML group are presented in Fig. 2A, B and C, respectively.

The results of the 24-h urine protein quantitation indicated that the urinary protein levels were similar among the 7 groups prior to L-NAME treatment. At the end of pregnancy, an increase in urinary protein was observed in animals that received injections of L-NAME $(\mathrm{P}<0.05)$; furthermore, the urinary protein content in the L-NAME-treated pregnant rats was significantly higher than that of the rats in the normal pregnancy group $(\mathrm{P}<0.05$; Fig. 2D). No difference in the amount of excreted urinary protein among the 9D LL, 10D LL and 10D ML groups was detected. At the same time, there were significant differences between 9D ML and 9D LL, and 10D LL and 10D ML. $(\mathrm{P}<0.05)$.

Adverse pregnancy outcomes in the L-NAME-treated groups. Adverse pregnancy outcomes were observed in the experimental groups, which may have resulted from high SBP or MAP. The pups of rats in the L-NAME-treated groups were significantly smaller than those of the rats in the control group, with a shorter crown-rump length and lower fetal weight $(\mathrm{P}<0.01)$. The placentas of the L-NAME-treated groups were smaller but heavier $(\mathrm{P}<0.01)$ and exhibited spherical edema compared with the placentas of the normal pregnancy rats. The placental diameter, placental weight, fetal weight and crown-rump length in the 9D ML and 10D ML groups were the most significantly different compared with those in the control group (Fig. 3A-D). However, the 10D ML group exhibited a more severe fetal absorption percentage than the 9D ML group (fetal malformations in $25 \%$ of fetuses).

The pregnant rats in the L-NAME-treated groups also exhibited significant changes in placental histology that are consistent with those reported in human preeclampsia patients. The placental trophoblast cells of the L-NAME-treated pregnant rats exhibited vacuolar aggregation (Fig. 3E-G).

Circulating sFlt-1 levels and the sFlt-1/PLGF ratio are significantly elevated in the pregnant rats injected with L-NAME. The plasma levels of sFlt-1 and PLGF in the pregnant rats of each group were measured by ELISA (Fig. 4). The concentration of sFlt-1 in the plasma of rats treated with L-NAME was increased, but no dose-dependent effect was observed. The plasma concentration of sFlt-1 in rats of the 9D ML group $(373.85 \pm 4.67 \mathrm{ng} / \mathrm{ml})$ and the 9D HL group $(346.30 \pm 8.23 \mathrm{ng} / \mathrm{ml})$ was significantly increased compared with that in rats of the control group $(281.02 \pm 5.77 \mathrm{ng} / \mathrm{ml}$; $\mathrm{P}<0.01$; Fig. 4A). The plasma concentration of PLGF in rats of the 9D ML group $(74.54 \pm 4.75 \mathrm{ng} / \mathrm{ml})$ and the 10D ML group $(80.69 \pm 5.14 \mathrm{ng} / \mathrm{ml})$ was significantly decreased compared with that in rats of the control group $(95.39 \pm 3.80 \mathrm{ng} / \mathrm{ml}$; $\mathrm{P}<0.01$; Fig. 4B). 


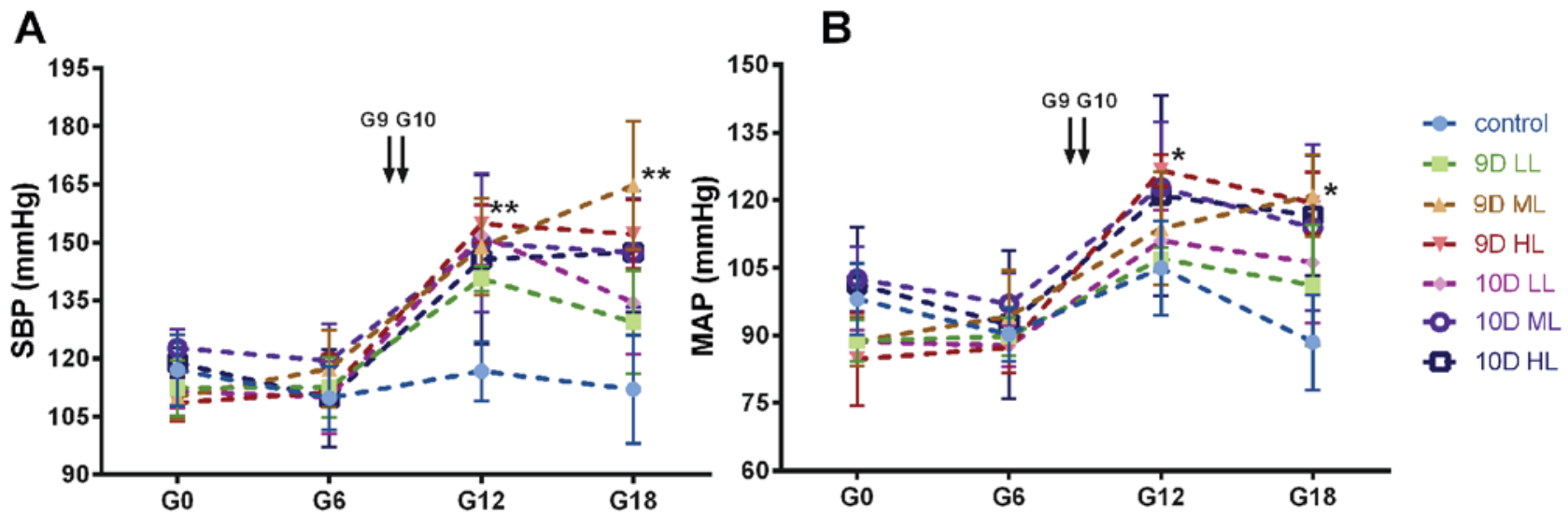

Figure 1. Blood pressure of pregnant rats was non-invasively measured with the CODA system (Kent Scientific, Torrington, CT, USA). The variation tendency of (A) SBP and (B) MAP during pregnancy in each group. ${ }^{*} \mathrm{P}<0.05 ;{ }^{* *} \mathrm{P}<0.01$ vs. the control group. Values are expressed as the mean $\pm 95 \%$ confidence interval. Groups: 9D LL, rats treated with low-dose L-NAME $(40 \mathrm{mg} / \mathrm{kg}$ body weight/day) starting from GD 9; 9D ML, rats treated with medium-L-NAME (75 mg/kg body weight/day) starting from GD 9; 9D HL, rats treated with high-dose L-NAME (125 mg/kg body weight/day) starting from GD 9; 10D LL, rats treated with low-dose L-NAME (40 mg/kg body weight/day) starting from GD 10;10D ML, rats treated with medium-L-NAME (75 mg/kg body weight/day) starting from GD 10; 10D HL, rats treated with high-dose L-NAME (125 mg/kg body weight/day) starting from GD 10; control, normal pregnancy group. GD, gestational day; L-NAME, N-nitro-L-arginine methyl ester; SBP, systolic blood pressure; MAP, mean arterial pressure.

The sFlt-1/PLGF ratio in the 9D ML group $(5.03 \pm 0.30 \mathrm{ng} / \mathrm{ml})$ and the 9D HL group $(4.17 \pm 0.43 \mathrm{ng} / \mathrm{ml})$ was significantly higher than that in the control group $(2.95 \pm 0.16 \mathrm{ng} / \mathrm{ml} ; \mathrm{P}<0.01$; Fig. 4C). However, no statistically significant differences were detected for the other groups.

Circulating oxidative stress levels are increased in rats after $L-N A M E$ treatment. The plasma levels of 8 -iso-PFG2 $\alpha$ and malondialdehyde were measured in pregnant rats (Fig. 5). The levels of 8 -iso-PFG2 $\alpha$ in the L-NAME-treated groups were higher than those in the control group $(96.71 \pm 1.12 \mathrm{ng} / \mathrm{ml})$, and a significant difference was present among the 9D ML $(115.29 \pm 7.67 \mathrm{ng} / \mathrm{ml}), 10 \mathrm{D} \mathrm{ML}(118.26 \pm 22.69 \mathrm{ng} / \mathrm{ml})$ and 10D HL groups $(121.44 \pm 9.82 \mathrm{ng} / \mathrm{ml} ; \mathrm{P}<0.01)$. However, the levels of 8 -iso-PFG2 $\alpha$ in the other three experimental groups were not significantly different from those in the control group (Fig. 5A).

MDA detection in the plasma of pregnant rats also suggested that the level of oxidative stress in the L-NAME treatment groups was higher than that in the control group. The plasma MDA level of the 9D ML group $(120.72 \pm 6.13 \mathrm{ng} / \mathrm{ml})$, 9D HL group $(103.85 \pm 6.01 \mathrm{ng} / \mathrm{ml})$ and 10D HL group $(94.44 \pm 7.89 \mathrm{ng} / \mathrm{ml})$ was significantly higher than that of the control group $(72.82 \pm 9.44 \mathrm{ng} / \mathrm{ml} ; \mathrm{P}<0.01$; Fig. 5B).

L-NAME-treated pregnant rats exhibit a more severe inflammatory reaction. The pathogenesis of preeclampsia involves inflammation, oxidative stress, endothelial injury and numerous other factors. In the present study, the BioLegend multifactor flow assay was used to detect the plasma levels of TNF- $\alpha$, IL-17A, MCP-1 and IL-1 $\beta$ in all groups (Fig. 6).

A significant difference in the levels of MCP-1 compared with that in the control group $(80.64 \pm 25.91 \mathrm{ng} / \mu \mathrm{l})$ was noted in the 9D ML group $(195.55 \pm 13.97 \mathrm{ng} / \mu \mathrm{l})$ and the 10D HL group $(173.73 \pm 22.92 \mathrm{ng} / \mu 1 ; \mathrm{P}<0.05$; Fig. 6A). However, there was no difference in the expression levels of TNF- $\alpha$ among those groups (Fig. 6B). The expression levels of IL-17A in all experimental groups except for the 10D LL group $(6.64 \pm 0.57 \mathrm{ng} / \mu \mathrm{l})$ were significantly increased compared with those in the control group (7.08 $\pm 1.50 \mathrm{ng} / \mu \mathrm{l} ; \mathrm{P}<0.05$; Fig. $6 \mathrm{C})$. However, there was no difference in the expression levels of IL- $1 \beta$ among those groups (Fig. 6D). Collectively, the results of the flow cytometry assay indicated that the levels of certain circulating inflammatory factors in pregnant rats were increased.

Changes of circulating $N O$ levels in rats after L-NAME treatment. The effects of different treatment regimens with L-NAME on the levels of NO in the circulation of pregnant rats in each group were determined by serum analysis towards the end of pregnancy (GD 20). The NO levels in the pregnant rats in each of the L-NAME-treated groups were significantly decreased compared with those in the control group $(57.83 \pm 1.53 \mu \mathrm{g} / \mathrm{ml} ; \mathrm{P}<0.01)$, except for those in the 10D LL group (54.49 $\pm 2.94 \mu \mathrm{g} / \mathrm{ml}$; Fig. 7).

\section{Discussion}

The L-NAME-induced SD rat model of preeclampsia developed in the present study may be a promising means of investigating the pathogenesis of preeclampsia and evaluating therapies for this condition. Rats treated with L-NAME exhibited elevated $\mathrm{BP}$, increased urinary albumin excretion, severe endotheliosis, mesangial expansion and increased sFlt-1/PLGF ratios.

Preeclampsia is a serious complication in pregnancy. Its major symptoms include high $\mathrm{BP}$, proteinuria, endothelial dysfunction, glomerular vascular endothelial cell proliferation and trophoblast cell infiltration. However, the pathogenesis of preeclampsia has remained to be fully elucidated.

Animal models are commonly used to study the pathogenesis of preeclampsia and develop novel diagnostic methods and treatments. At present, a wide range of preeclampsia models exist, including the spontaneous preeclampsia model $(24,25)$, the NOS inhibition model (26), the angiogenesis factor imbalance model $(27,28)$, the oxygen deprivation model $(29,30)$, the immune response model $(31,32)$ and the chronic hypertension and vascular injury model $(33,34)$. However, an effective animal model is still required to simulate the complex features 

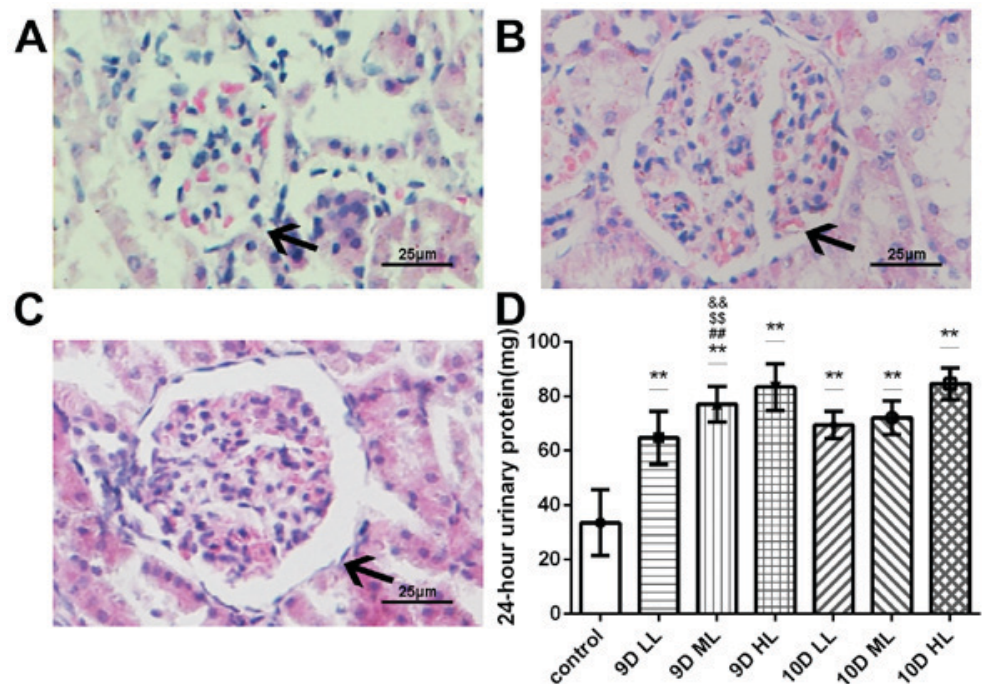

Figure 2. Changes of kidney structure and function in pregnant rats (GD20). (A-C) Histopathological images of glomeruli in pregnant rats of (A) the control group, (B) the 9D ML group and (C) the 10D ML group (H\&E; magnification, $\mathrm{x} 400$; scale bar, $25 \mu \mathrm{m}$ ). Black arrows indicate glomeruli. (D) The 24-h urine protein of the pregnant rats in GD20. ${ }^{* *} \mathrm{P}<0.05$ vs. control group; ${ }^{\# \#} \mathrm{P}<0.05$ 9D ML group vs. 9D LL group; ${ }^{\$ S} \mathrm{P}<0.05$ 9D ML group vs. 10D LL group; \&\& $\mathrm{P}<0.05$ 9D ML group vs. 10D ML group. Values are expressed as the mean \pm standard deviation. Groups: 9D LL, rats treated with low-dose L-NAME (40 mg/kg body weight/day) starting from at GD 9; 9D ML, rats treated with medium-L-NAME (75 mg/kg body weight/day) starting from GD 9; 9D HL, rats treated with high-dose L-NAME $(125 \mathrm{mg} / \mathrm{kg}$ body weight/day) starting from GD 9; 10D LL, rats treated with low-dose L-NAME (40 mg/kg body weight/day) starting from GD 10; 10D ML, rats treated with medium-L-NAME (75 mg/kg body weight/day) starting from GD 10; 10D HL, rats treated with high-dose L-NAME $(125 \mathrm{mg} / \mathrm{kg}$ body weight/day) starting from GD 10; control, normal pregnancy group. GD, gestational day; L-NAME, N-nitro-L-arginine methyl ester.

A

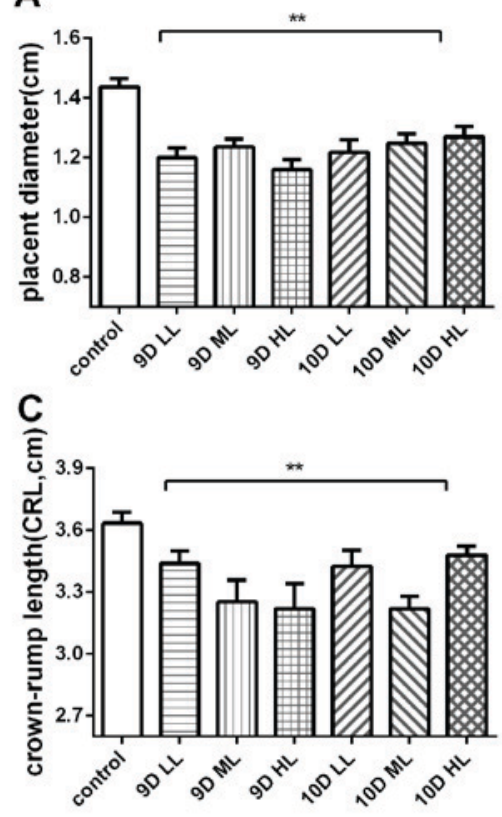

E

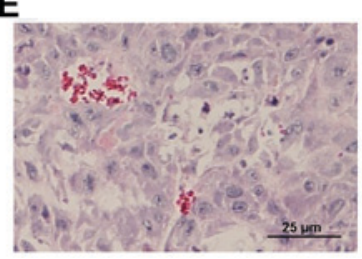

$\mathbf{F}$

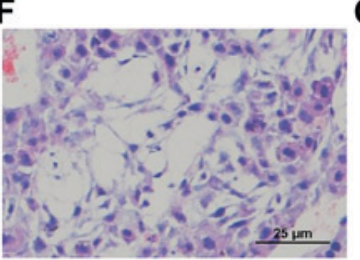

B

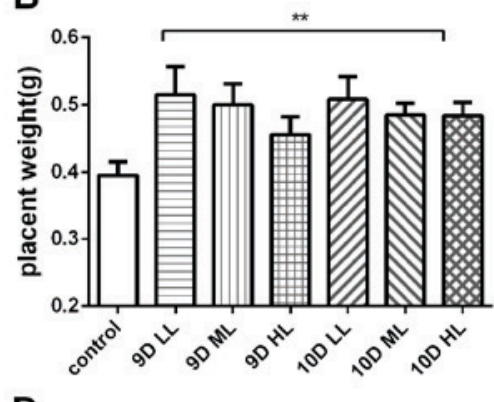

D

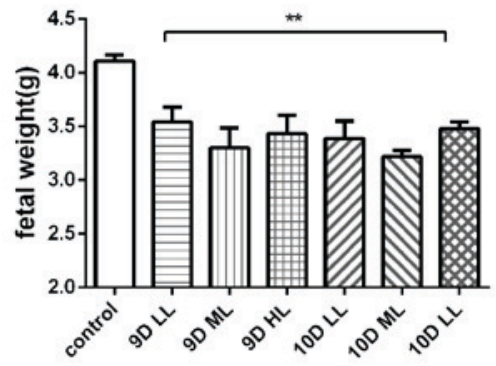

G

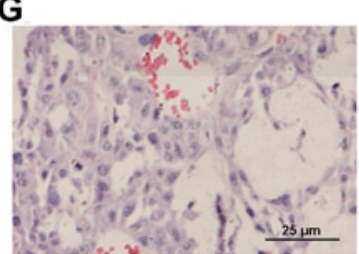

Figure 3. Pregnancy outcome and changes in placental trophoblast structure in the pregnant rats at the end of pregnancy (GD20). (A) Diameter of the placenta in each group. (B) Weight of the placenta in each group. (C) Crown-rump length in each group. (D) Fetal weight in each group. (E-G) Histopathological images of placental trophoblasts in pregnant rats of (E) the control group, (F) 9D ML group and (G) 10D ML group (H\&E staining; magnification, x400; scale bar, $25 \mu \mathrm{m}){ }^{* * *} \mathrm{P}<0.05$ vs. control group. Values are expressed as the mean \pm standard deviation. Groups: 9D LL, rats treated with low-dose L-NAME (40 mg/kg body weight/day) starting from GD 9; 9D ML, rats treated with medium-L-NAME (75 mg/kg body weight/day) starting from GD 9; 9D HL, rats treated with high-dose L-NAME (125 mg/kg body weight/day) starting from GD 9; 10D LL, rats treated with low-dose L-NAME (40 mg/kg body weight/day) starting from GD 10; 10D ML, rats treated with medium-L-NAME (75 mg/kg body weight/day) starting from GD 10; 10D HL, rats treated with high-dose L-NAME $(125 \mathrm{mg} / \mathrm{kg}$ body weight/day) starting from GD 10; control, normal pregnancy group. GD, gestational day; L-NAME, N-nitro-L-arginine methyl ester. 

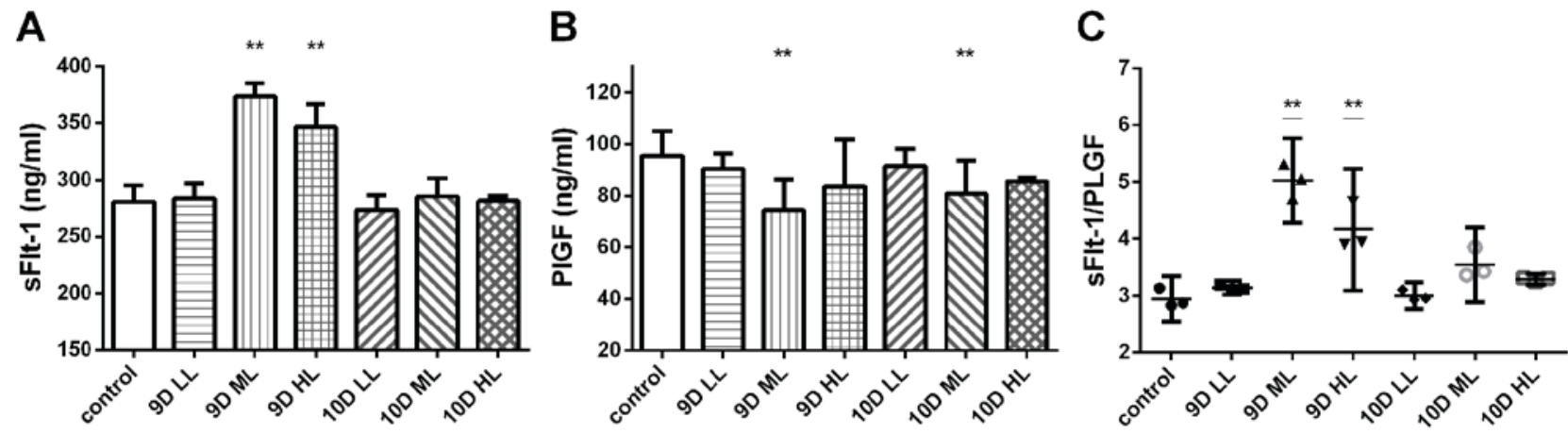

Figure 4. Plasma levels of sFlt-1 and PLGF in the pregnant rats in each group at the end of pregnancy (GD20) were determined by ELISA. (A) Circulating sFlt-1 levels. (B) Circulating PLGF levels. (C) Circulating sFlt-1/PLGF ratio in the pregnant rats. ${ }^{* *} \mathrm{P}<0.05$ vs. control group. Values are expressed as the mean \pm standard deviation. Groups: 9D LL, rats treated with low-dose L-NAME (40 mg/kg body weight/day) starting from GD 9; 9D ML, rats treated with medium-L-NAME (75 mg/kg body weight/day) starting from GD 9; 9D HL, rats treated with high-dose L-NAME (125 mg/kg body weight/day) starting from GD 9; 10D LL, rats treated with low-dose L-NAME ( $40 \mathrm{mg} / \mathrm{kg}$ body weight/day) starting from GD 10; 10D ML, rats treated with medium-L-NAME (75 mg/kg body weight/day) starting from GD 10; 10D HL, rats treated with high-dose L-NAME (125 mg/kg body weight/day) starting from GD 10; control, normal pregnancy group. GD, gestational day; L-NAME, N-nitro-L-arginine methyl ester; sFlt-1, soluble fms-like tyrosine kinase-1; PLGF, placental growth factor.

A

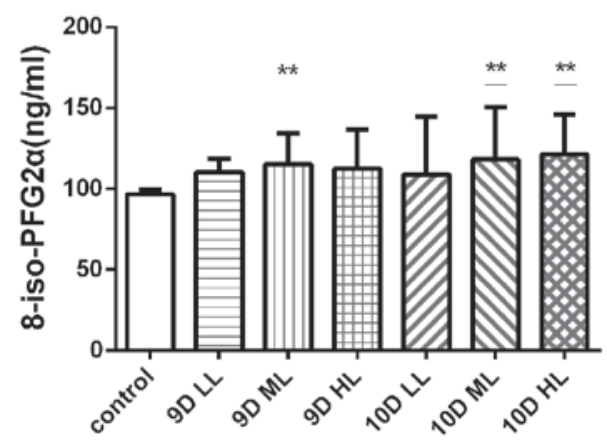

B

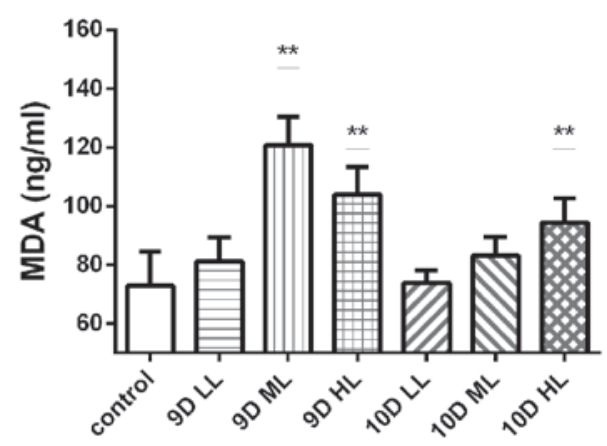

Figure 5. Levels of 8-iso-PFG2 $\alpha$ and MDA in the plasma of pregnant rats in each group at the end of pregnancy (GD20) were measured by ELISA or thiobarbituric acid reactive substances. (A) Circulating 8 -iso-PGF2 $\alpha$ levels in pregnant rats. (B) Circulating MDA levels in pregnant rats. ${ }^{* *} \mathrm{P}<0.05$ vs. control group. Values are expressed as the mean \pm standard deviation. Groups: 9D LL, rats treated with low-dose L-NAME (40 mg/kg body weight/day) starting from GD 9; 9D ML, rats treated with medium-L-NAME (75 mg/kg body weight/day) starting from GD 9; 9D HL, rats treated with high-dose L-NAME (125 mg/kg body weight/day) starting from GD 9; 10D LL, rats treated with low-dose L-NAME (40 mg/kg body weight/day) starting from GD 10; 10D ML, rats treated with medium-L-NAME (75 mg/kg body weight/day) starting from GD 10; 10D HL, rats treated with high-dose L-NAME (125 mg/kg body weight/day) starting from GD 10; control, normal pregnancy group. GD, gestational day; L-NAME, N-nitro-L-arginine methyl ester; MDA, malondialdehyde; PGF2, prostaglandin F2.

of preeclampsia. The present study aimed to explore the ideal dosing and timing of L-NAME administration to pregnant rats for the establishment of a model of preeclampsia.

During pregnancy, NO synthesis and its release in endothelial cells have an important role in vascular relaxation and the regulation of vascular tension (35). Chronic inhibition of NO production increases the $\mathrm{BP}$ in a volume-dependent manner, and the associated physiological and pathological characteristics are similar to those of primary hypertension (36). In addition, it is known that acute inhibition of NO biosynthesis by the administration of L-NAME results in arterial hypertension and vasoconstriction. Therefore, in the present study a novel preeclampsia animal model was constructed via subcutaneous injection of the NOS inhibitor L-NAME into rats starting at mid-pregnancy. It was revealed all groups of pregnant rats injected with L-NAME except for those in the 10D LL group had significantly lower circulating NO levels than those in the control group. It is suggested that L-NAME treatment inhibits NOS in pregnant rats and induces the corresponding changes in preeclampsia $(37,38)$.
The present study indicated that L-NAME successfully induced preeclampsia in pregnant rats. The dosing and timing of L-NAME injection are of great importance, as late drug administration or low doses cannot induce a statistically significant difference in BP to simulate the mid-pregnancy $\mathrm{BP}$ increases observed in preeclampsia patients. In the present study, L-NAME was injected from GD 9 or GD 10. Based on an extensive literature search (17,19), L-NAME at 40, 75 and $125 \mathrm{mg} / \mathrm{kg} /$ day was used to induce preeclampsia in pregnant rats. As expected, the SBP and MAP were elevated in L-NAME-induced hypertensive rats, and the pressure difference was $30 \mathrm{mmHg}$ after the administration of L-NAME to the 9D ML, 9D HL, 10D ML and 10D HL groups from GD12. The difference in BP was observed until delivery at GD 20, particularly in the 9D ML group. Furthermore, the rats in all groups except the 9D ML group exhibited normal pregnancy patterns, with a decrease in SBP and MAP during late pregnancy, whereas the rats in the 9D ML group did not exhibit any drop in BP; rather, these rats exhibited a steady increase in BP during pregnancy. Thus, the results of the present study 
A

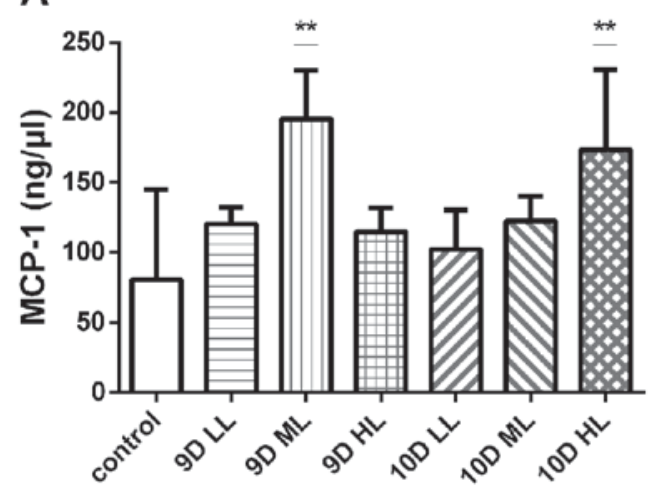

B

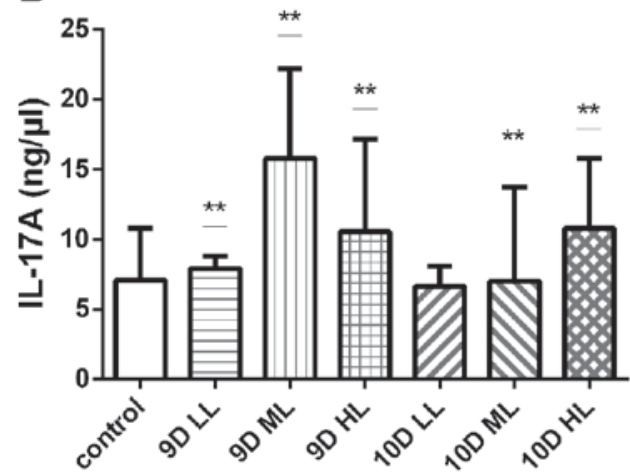

C

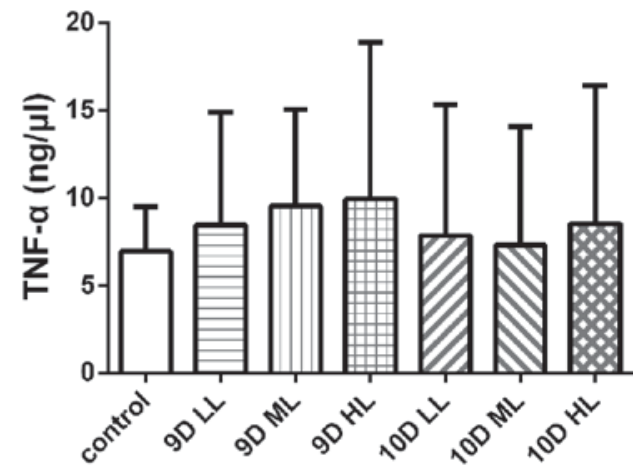

D

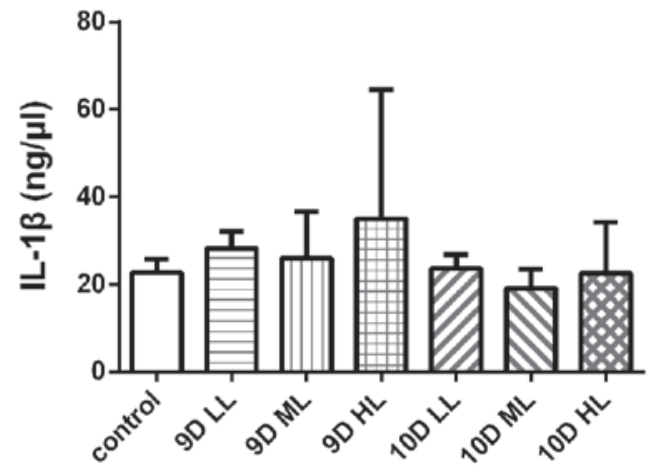

Figure 6. Levels of inflammatory factors in the plasma of pregnant rats in each group at the end of pregnancy (GD20) were measured using the BioLegend multi-factor flow assay. (A) Circulating MCP-1, (B) IL-17A, (C) TNF- $\alpha$ and (D) IL- $1 \beta$ levels. ${ }^{* *} \mathrm{P}<0.05$ vs. control group. Values are expressed as the mean \pm standard deviation. Groups: 9D LL, rats treated with low-dose L-NAME (40 mg/kg body weight/day) starting from GD 9; 9D ML, rats treated with medium-L-NAME (75 mg/kg body weight/day) starting from GD 9; 9D HL, rats treated with high-dose L-NAME (125 mg/kg body weight/day) starting from GD 9; 10D LL, rats treated with low-dose L-NAME (40 mg/kg body weight/day) starting from GD 10; 10D ML, rats treated with medium-L-NAME (75 mg/kg body weight/day) starting from GD 10; 10D HL, rats treated with high-dose L-NAME (125 mg/kg body weight/day) starting from GD 10; control, normal pregnancy group. GD, gestational day; L-NAME, N-nitro-L-arginine methyl ester; TNF, tumor necrosis factor; IL, interleukin; MCP, monocyte chemoattractant protein.

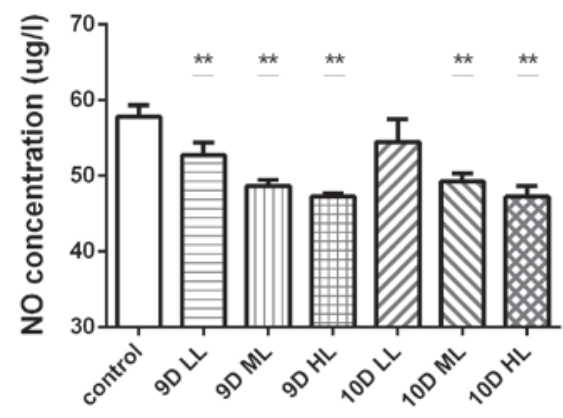

Figure 7. Levels of circulating NO in pregnant rats in each group at the end of pregnancy (GD20) were measured by nitrate reductase method. ${ }^{* *} \mathrm{P}<0.05$ vs. control group. Values are expressed as the mean \pm standard deviation. Groups: 9D LL, rats treated with low-dose L-NAME (40 mg/kg body weight/day) starting from GD 9; 9D ML, rats treated with medium-L-NAME (75 mg/kg body weight/day) starting from GD 9; 9D HL, rats treated with high-dose L-NAME (125 mg/kg body weight/day) starting from GD 9; 10D LL, rats treated with low-dose L-NAME (40 mg/kg body weight/day) starting from GD 10; 10D ML, rats treated with medium-L-NAME ( $75 \mathrm{mg} / \mathrm{kg}$ body weight/day) starting from GD 10; 10D HL, rats treated with high-dose L-NAME $(125 \mathrm{mg} / \mathrm{kg}$ body weight/day) starting from GD 10; control, normal pregnancy group. GD, gestational day; L-NAME, N-nitro-L-arginine methyl ester; NO, nitric oxide.

indicate that the establishment of a pre-eclampsia model for SD pregnant rats (starting from the 9th day of pregnancy with a continuous subcutaneous injection of $75 \mathrm{mg} / \mathrm{kg} / \mathrm{day}$ ) is a promising solution. Compared with the previous study (37), the current study assessed L-NAME dosage, time and the effect of L-NAME in combination. Following L-NAME treatment, significant changes in maternal urine protein content, the placenta, the kidneys and the blood vessels were observed in pregnant rats with preeclampsia, particularly those in the D9 ML, 9D HL and 10D HL groups. In preeclampsia, maternal uterine spiral artery remodeling disorders lead to placental ischemia and placenta angiogenesis imbalances, the levels of sFlt-1 are elevated and the levels of PLGF, a subtype of vascular endothelial growth factor, are decreased (39). In the present study, the plasma concentration of sFlt-1 in rats of the 9D ML and 9D HL groups was significantly increased compared with that in rats of the control group, and the concentration of PLGF in rats of the 9D ML and 10D ML groups was significantly decreased compared with that in rats of the control group. The ratio of sFlt-1 to PLGF in the 9D ML and 9D HL groups was significantly higher than that in the control group. The above results indicated that L-NAME intervention on the 9th day of pregnancy is more suitable to simulate pre-eclampsia symptoms. In addition, it has been reported that the mechanism of urinary protein aggravation may be associated with the release of circulating factors, including TNF- $\alpha$ or the anti-angiogenic factor sFlt-1, but this observation requires further study (32).

According to the present results, L-NAME-induced preeclampsia-like pregnant rats also exhibited oxidative stress and inflammatory changes. The plasma levels of 8-iso-PFG $2 \alpha$ 
were increased in all L-NAME-treated groups, and significant differences from the control group were observed in the 9D ML, 10D ML and 10D HL groups. Similarly, the plasma levels of MDA in the pregnant rats after L-NAME treatment were substantially increased. As observed in the development of human preeclampsia, L-NAME-treated pregnant rats exhibited oxidative stress, which may be one of the causes of preeclampsia or may lead to more serious premenstrual symptoms (40). MCP-1 is a secreted single-chain protein and a member of the chemokine family; as one of the major chemokines, MCP-1 induces chemotaxis via the $\mathrm{G}$ protein-coupled receptor pathway, as well as macrophage migration and infiltration. MCP-1 releases chemokines to recruit monocytes into the endodermis, which may be transformed into macrophages and phagocytes with selectivity for oxidized low-density lipoprotein, and are then transformed into foam cells. MCP-1 also participates in cell growth, metabolism and apoptosis. In the present study, the plasma levels of MCP-1 were identified to be elevated, indicating that an excessive inflammatory reaction occurs in the rats with preeclampsia. Excess macrophages may infiltrate the decidua, damage the vascular endothelial cells, and prevent intravascular trophoblast invasion and vascular remodeling in the placenta; at the same time, MCP-1 may also affect inflammatory cell infiltration at the maternal-fetal interface, and cause abnormal placental trophoblastic cells, increases in apoptosis and acute atherosclerosis of the small uterine spiral artery, all of which induces the occurrence of preeclampsia.

IL-17A is a cytokine produced by numerous cell types, including T-helper type 17 (Th17) cells (41). IL-17A regulates the expression of cytokines, chemokines and adhesion molecules in the microenvironment through distinct pathways, increases the levels of inflammatory cells, particularly neutrophils, and induces an inflammatory effect (42). IL-17A is involved in inflammatory diseases. High levels of IL-17A, Th1/Th2 drift and the pro-inflammatory response are inseparable, and may interact with a variety of cytokines to enhance small vascular inflammation in the placenta. Elevated IL-17A also has a marked ability to raise neutrophil levels, induce the release of active substances to damage endothelial cells and at the same time, to generate a large number of oxygen free radicals to cause damage to vascular endothelial cells, increase the permeability of blood vessels, and increase BP in preeclampsia, vasospasm, edema, proteinuria and a series of pathological factors associated with various clinical manifestations. Multifactor flow detection is an effective method for detecting trace factors in tissues, plasma, cell supernatants and other samples. The present indicated that the levels of IL-17A and MCP-1 were elevated in the plasma of the experimental groups, suggesting that an inflammatory response occurs in the L-NAME-induced preeclampsia-like rat model.

Although numerous studies of L-NAME induced animal models of preeclampsia have been established, due to the different descriptions of this method, the present study aimed to optimize the timing and dosing as approaches of molding $(8,38)$. The present study further indicated that oxidative stress and inflammation have important roles in the development of preeclampsia, which is based on numerous previous studies $(43,44)$. When assessing the clinical manifestations (hypertension, proteinuria, and maternal and offspring outcomes) and pathological changes (alterations in the pathological structure of placenta and kidney) of preeclampsia, certain molecules that represent significant oxidative stress and inflammatory responses were assessed and compared. The similarity between the animal disease model and real disease was further verified, which is in favor of the experiment aimed to establish the model. The present experiments led to the successful development of a model that exhibits preeclampsia-like characteristics, including phenotype (gestational hypertension and proteinuria), pregnancy outcome, birth effects in offspring and serological markers in pregnant SD rats. Due to the sustained action of the NOS inhibitor L-NAME, a number of hemodynamic changes were observed in the pregnant rats, including placental and renal ischemia, as well as hypoxia symptoms, oxidative stress and an intensified inflammatory response. Therefore, the L-NAME-induced preeclampsia model provides a powerful tool with which to investigate the pathogenesis of preeclampsia. The rat model of preeclampsia allows for the analysis of time-dependent changes in the placenta, vasculature and kidney throughout pregnancy, as well as the discovery of novel biomarkers that are present during early pregnancy. The construction of this model was simple and it may be used to study neonatal markers, the pathophysiological mechanisms of preeclampsia and drug targets for its prevention. The offspring with birth defects and dysplasia may also be used for further study of preeclampsia progeny. It was noted that this model may lead to fetal malformations (limb reduction), which may be due to the side effects of NOS inhibition by L-NAME. Of note, the present study had a certain limitation. The obtainment of blood is required for the detection of plasma markers, and frequent blood extraction may have affected the experiment; furthermore, the availability of kidney and placenta tissue is limited by the amount of experimental animals. Therefore, the present study did not perform any other relevant tests on GD 14 and GD 18. This limitation of the present study will be further improved in future experiments. In the future, animal models will continue to enhance the current understanding of the physiological and pathological conditions of trophoblast cell differentiation and invasion during pregnancy.

\section{Acknowledgements}

Not applicable.

\section{Funding}

The study was supported by Tianjin Science and Technology Major Projects (grant nos. 16ZXMJSY00130 and 15ZXJZSY00010).

\section{Availability of data and materials}

The datasets used and/or analyzed during the current study are available from the corresponding author on reasonable request.

\section{Authors' contributions}

WS and HL participated in all experiments, and they were major contributor in writing the manuscript. HG participated in most experiments except TBARS, ELISA and LEGENDplexTM. 
$\mathrm{MZ}$ and $\mathrm{XN}$ participated in the quality monitoring of all experimental methods and results. YM participated in LEGENDplexTM. XZ participated in blood pressure monitoring. WC participated in tissue collection. GY participated in pathological staining of placenta and kidney. MW analyzed and interpreted all experimental data. NY and YL participated in the experimental design and guided the experiment. All authors read and approved the final manuscript.

\section{Ethical approval and consent to participate}

The present study was approved by the Ethics Committee of the Logistics University of the Chinese People's Armed Police Forces (Tianjin, China).

\section{Consent for publication}

Not applicable.

\section{Competing interests}

The authors declare no potential competing interests with respect to the research, authorship and/or publication of this article.

\section{References}

1. American College of Obstetricians and Gynecologists; Task Force on Hypertension in Pregnancy: Hypertension in pregnancy. Report of the American College of Obstetricians and Gynecologists' Task Force on Hypertension in Pregnancy. Obstet Gynecol 122: 1122-1131, 2013.

2. Al-Jameil N, Aziz Khan F, Fareed Khan M and Tabassum H: A brief overview of preeclampsia. J Clin Med Res 6: 1-7, 2014.

3. Wang A, Rana S and Karumanchi SA: Preeclampsia: The role of angiogenic factors in its pathogenesis. Physiology (Bethesda) 24 147-158, 2009.

4. ACOG Committee on Obstetric Practice: ACOG practice bulletin. Diagnosis and management of preeclampsia and eclampsia. Number 33, January 2002. American College of Obstetricians and Gynecologists. Int J Gynaecol Obstet 77: 67-75, 2002.

5. Khan KS, Wojdyla D, Say L, Gülmezoglu AM and Van Look PF: WHO analysis of causes of maternal death: A systematic review. Lancet 367: 1066-1074, 2006.

6. Steegers EA, von Dadelszen P, Duvekot JJ and Pijnenborg R: Pre-eclampsia. Lancet 376: 631-644, 2010.

7. European Society of Gynecology (ESG); Association for European Paediatric Cardiology (AEPC); German Society for Gender Medicine (DGesGM), Regitz-Zagrosek V, Blomstrom Lundqvist C, Borghi C, Cifkova R, Ferreira R, Foidart JM, Gibbs JS, et al: ESC Guidelines on the management of cardiovascular diseases during pregnancy: The Task Force on the Management of Cardiovascular Diseases during Pregnancy of the European Society of Cardiology (ESC). Eur Heart J 32: 3147-3197, 2011

8. Soobryan N, Murugesan S, Phoswa W, Gathiram P, Moodley J and Mackraj I: The effects of sildenafil citrate on uterine angiogenic status and serum inflammatory markers in an L-NAME rat model of pre-eclampsia. Eur J Pharmacol 795: 101-107, 2017.

9. Jim B and Karumanchi SA: Preeclampsia: Pathogenesis, prevention, and long-term complications. Semin Nephrol 37: 386-397, 2017.

10. Lisonkova S and Joseph KS: Incidence of preeclampsia: Risk factors and outcomes associated with early- versus late-onset disease. Am J Obstet Gynecol 209: 544.e1-544.e12, 2013.

11. Hladunewich $M$, Karumanchi SA and Lafayette $R$ : Pathophysiology of the clinical manifestations of preeclampsia. Clin J Am Soc Nephrol 2: 543-549, 2007.

12. Roberts JM and Gammill HS: Preeclampsia: Recent insights. Hypertension 46: 1243-1249, 2005.
13. Lam C, Lim KH and Karumanchi SA: Circulating angiogenic factors in the pathogenesis and prediction of preeclampsia. Hypertension 46: 1077-1085, 2005.

14. Chaiworapongsa T, Chaemsaithong P, Yeo L and Romero R: Pre-eclampsia part 1: Current understanding of its pathophysiology. Nat Rev Nephrol 10: 466-480, 2014.

15. Powe CE, Levine RJ and Karumanchi SA: Preeclampsia, a disease of the maternal endothelium: The role of antiangiogenic factors and implications for later cardiovascular disease. Circulation 123: 2856-2869, 2011.

16. Zeisler H, Hund $M$ and Verlohren S: The sFlt-1: PlGF ratio in women with suspected preeclampsia. N Engl J Med 374: 1785-1786, 2016.

17. Motta C, Grosso C,Zanuzzi C, Molinero D, Picco N, Bellingeri R, Alustiza F, Barbeito C, Vivas A and Romanini MC: Effect of Sildenafil on Pre-Eclampsia-Like Mouse Model Induced By L-Name. Reprod Domest Anim 50: 611-616, 2015.

18. Souza CO, Peraçoli MT, Weel IC, Bannwart CF, Romão M, Nakaira-Takahagi E, Medeiros LT, Silva MG and Peraçoli JC: Hepatoprotective and anti-inflammatory effects of silibinin on experimental preeclampsia induced by L-NAME in rats. Life Sci 91: 159-165, 2012.

19. Liu W, Qiao F, Liu H, Gong X, Shi X, Li Y and Wu Y: Low molecular weight heparin improves proteinuria in rats with L-NAME induced preeclampsia by decreasing the expression of nephrin, but not podocin. Hypertens Pregnancy 34: 24-35, 2015.

20. Rowsell HC: Program and objectives of the Canadian Council on Animal Care. Lab Anim Sci 37: 24-27, 1987.

21. Song Y, Shi MM, Zhang YY, Mo XD, Wang Y, Zhang XH, $\mathrm{Xu}$ LP, Huang XJ and Kong Y: Abnormalities of the bone marrow immune microenvironment in patients with prolonged isolated thrombocytopenia after allogeneic hematopoietic stem cell transplantation. Biol Blood Marrow Transplant 23: 906-912, 2017.

22. Chatziandreou N, Farsakoglu Y, Palomino-Segura M, D'Antuono R, Pizzagalli DU, Sallusto F, Lukacs-Kornek V, Uguccioni M, Corti D, Turley SJ, et al: Macrophage death following influenza vaccination initiates the inflammatory response that promotes dendritic cell function in the draining lymph node. Cell Rep 18: 2427-2440, 2017.

23. Zhu Y, Paniccia A, Schulick AC, Chen W, Koenig MR, Byers JT, Yao S, Bevers S and Edil BH: Identification of CD112R as a novel checkpoint for human T cells. J Exp Med 213: 167-176, 2016.

24. Gille JH, Moore DG and Sedgwick CJ: Placental infarction: A sign of pre-eclampsia in a patas monkey (Erythrocebus patas). Lab Anim Sci 27: 119-121, 1977.

25. Rogers JB, Klein R and Blumenthal HT: Placental permeability in a toxemia-susceptible strain of guinea pigs. Am J Obstet Gynecol 88: 495-501, 1964.

26. Diket AL,Pierce MR,Munshi UK, VoelkerCA,Eloby-Childress S, Greenberg SS, Zhang XJ, Clark DA and Miller MJ: Nitric oxide inhibition causes intrauterine growth retardation and hind-limb disruptions in rats. Am J Obstet Gynecol 171: 1243-1250, 1994.

27. Kumasawa K, Ikawa M, Kidoya H, Hasuwa H, Saito-Fujita T, Morioka Y, Takakura N, Kimura T and Okabe M: Pravastatin induces placental growth factor (PGF) and ameliorates preeclampsia in a mouse model. Proc Natl Acad Sci USA 108: 1451-1455, 2011.

28. Venkatesha S, Toporsian M, Lam C, Hanai J, Mammoto T, Kim YM, Bdolah Y, Lim KH, Yuan HT, Libermann TA, et al: Soluble endoglin contributes to the pathogenesis of preeclampsia. Nat Med 12: 642-649, 2006.

29. Tal R, Shaish A, Barshack I, Polak-Charcon S, Afek A, Volkov A Feldman B, Avivi C and Harats D: Effects of hypoxia-inducible factor-1alpha overexpression in pregnant mice: Possible implications for preeclampsia and intrauterine growth restriction. Am J Pathol 177: 2950-2962, 2010.

30. Kanasaki K, Palmsten K, Sugimoto H, Ahmad S, Hamano Y, Xie L, Parry S, Augustin HG, Gattone VH, Folkman J, et al: Deficiency in catechol-O-methyltransferase and 2-methoxyoestradiol is associated with pre-eclampsia. Nature 453: 1117-1121, 2008.

31. Kupferminc MJ, Peaceman AM, Wigton TR, Rehnberg KA and Socol ML: Tumor necrosis factor-alpha is elevated in plasma and amniotic fluid of patients with severe preeclampsia. Am J Obstet Gynecol 170: 1752-1757; discussion 1757-1759, 1994.

32. LaMarca BB, Bennett WA, Alexander BT, Cockrell K and Granger JP: Hypertension produced by reductions in uterine perfusion in the pregnant rat: Role of tumor necrosis factor-alpha. Hypertension 46: 1022-1025, 2005. 
33. Wenzel K, Rajakumar A, Haase H, Geusens N, Hubner N, Schulz H, Brewer J, Roberts L, Hubel CA, Herse F, et al: Angiotensin II type 1 receptor antibodies and increased angiotensin II sensitivity in pregnant rats. Hypertension 58: 77-84, 2011.

34. Takimoto-Ohnishi E, Saito T, Ishida J, Ohnishi J, Sugiyama F, Yagami K and Fukamizu A: Differential roles of renin and angiotensinogen in the feto-maternal interface in the development of complications of pregnancy. Mol Endocrinol 19: 1361-1372, 2005.

35. Baksu B, Davas I, Baksu A, Akyol A and Gulbaba G: Plasma nitric oxide, endothelin-1 and urinary nitric oxide and cyclic guanosine monophosphate levels in hypertensive pregnant women. Int J Gynaecol Obstet 90: 112-117, 2005.

36. Vechoropoulos M, Ish-Shalom M, Shaklai S, Sack J, Stern N and Tordjman KM: The proatherogenic effect of chronic nitric oxide synthesis inhibition in ApoE-Null mice is dependent on the presence of PPAR $\alpha$. PPAR Res 2014: 124583, 2014.

37. Ribeiro MO, Antunes E, de Nucci G, Lovisolo SM and Zatz R: Chronic inhibition of nitric oxide synthesis. A new model of arterial hypertension. Hypertension 20: 298-303, 1992.

38. Wang Y,Zhang F, Liu Y, Yin S, Pang X, Li Z and Wei Z: Nebivolol alleviates aortic remodeling through eNOS upregulation and inhibition of oxidative stress in 1-NAME-induced hypertensive rats. Clin Exp Hypertens 39: 628-639, 2017.

39. Zeisler H,LlurbaE, Chantraine F, Vatish M, Staff AC, Sennström M, Olovsson M, Brennecke SP, Stepan H, Allegranza D, et al: Predictive value of the sFlt-1:PIGF ratio in women with suspected preeclampsia. N Engl J Med 374: 13-22, 2016.
40. Amaral TAS, Ognibene DT, Carvalho LCRM, Rocha APM, Costa CA, Moura RS and Resende AC: Differential responses of mesenteric arterial bed to vasoactive substances in L-NAME-induced preeclampsia: Role of oxidative stress and endothelial dysfunction. Clin Exp Hypertens 40: 126-135, 2018.

41. Cornelius DC, Hogg JP, Scott J, Wallace K, Herse F, Moseley J, Wallukat G, Dechend R and LaMarca B: Administration of interleukin-17 soluble receptor C suppresses TH17 cells, oxidative stress, and hypertension in response to placental ischemia during pregnancy. Hypertension 62: 1068-1073, 2013.

42. Miossec P and Kolls JK: Targeting IL-17 and TH17 cells in chronic inflammation. Nat Rev Drug Discov 11: 763-776, 2012.

43. Guerby P, Vidal F, Garoby-Salom S, Vayssiere C, Salvayre R Parant $\mathrm{O}$ and Negre-Salvayre A: Oxidative stress and preeclampsia: A review. Gynecol Obstet Fertil 43: 751-756, 2015 (In French).

44. Walker JJ: Inflammation and preeclampsia. Pregnancy Hyperte-ns 1: 43-47, 2011.

This work is licensed under a Creative Commons Attribution-NonCommercial-NoDerivatives 4.0 International (CC BY-NC-ND 4.0) License. 